\title{
Synthesis of Chalcone Derivatives Containing Furan or/and Pyran Ring as Neuraminidase Inhibitors
}

\author{
CHEN Aiyu ${ }^{1}$, LIANG Yongdong ${ }^{1}$, YE Jiao ${ }^{1}$, HU Aixi $^{1}{ }^{*}$, LIAN Wenwen $^{2}$, \\ LIU Ailin $^{2 *}$ and DU Guanhua ${ }^{2}$ \\ 1. College of Chemistry and Chemical Engineering, Hunan University, Changsha 410082, P. R. China; \\ 2. Institute of Materia Medica, Chinese Academy of Medical Sciences \& Peking Union Medical College, \\ Beijing 100050, P. R. China
}

\begin{abstract}
Twenty-seven novel chalcone derivatives were designed and synthesized as neuraminidase(NA) inhibitors. A concise suitable synthetic strategy was employed in the target compounds' synthesis with relatively high yields. The synthesized compounds were evaluated for their inhibitory activities against the NA of influenza A virus in vitro. The results show that compound $9 \mathrm{~b}$ possesses the most potent NA inhibitory activity. Structure-activity relationship studies indicate that the chalcone system and hydrogen bond donor substituent are significant for the NA inhibitory activity. And the chalcone derivatives containing pyran ring have better NA inhibitory activity than those without the pyran ring. In addition, molecular docking studies reveal that compounds $9 \mathrm{~b}$ and $\mathbf{9 u}$ are in the good binding mode with Zanamivir binding sites. This study indicates that compound $9 \mathrm{~b}$ could be selected as a potent compound for further structural optimization and development of novel NA inhibitors.
\end{abstract}

Keywords Chalcone derivative; Neuraminidase inhibitor; Molecular docking

\section{Introduction}

The influenza virus infection is one of the most serious threats to worldwide public health and causes large economic and property losses ${ }^{[1,2]}$. The pandemic of influenza of H3N2 mutation caused more than 300 deaths in Hong Kong in 2017. At present, two classes of anti-influenza drugs that have been approved to treat influenza are M2 ion channel protein inhibitors and neuraminidase(NA) inhibitors ${ }^{[3-5]}$. Amantadine and rimantadine are M2 ion channel inhibitors ${ }^{[6,7]}$. Zanamivir, Oseltamivir, Peramivir and Laninamvir are NA inhibitors ${ }^{[8]}$. Compared with M2 inhibitors, NA inhibitors have improved security and availability for the treatment of influenza ${ }^{[9]}$. However, current treatment has been limited due to the emergence of resistant viral strains and strong side effects ${ }^{[10,11]}$. Therefore, it is of great importance to develop novel NA inhibitors, which contain a new scaffold with higher efficacy and enhanced tolerability against resistant viral strains ${ }^{[12]}$.

Natural products are a continuing source of novel drug leads ${ }^{[13-15]}$. Sulfuretin with furan moiety has been demonstrated to be a potent NA inhibitor with an $\mathrm{IC}_{50}$ (half maximal inhibitory concentration) value of $29.6 \mu \mathrm{mol} / \mathrm{L}^{[16]}$. 4-Hydroxyderricin(Fig.1), an alkylated chalcone from Angelica keiskei, showed potent NA inhibition with an $\mathrm{IC}_{50}$ value of 42.1 $\mu \mathrm{mol} / \mathrm{L}^{[17]}$. Kumatakenin(Fig.1), isolated from the root of Glycyrrhiza uralensis, showed potent NA inhibitory activity with an $\mathrm{IC}_{50}$ value of $36.4 \mu \mathrm{mol} / \mathrm{L}^{[18]}$. All these natural com- pounds contained chalcone moiety, indicating that chalcone moiety is critical for NA inhibitory activity. Moreover, incorporation of a furan ring into the chalcone scaffold seems to be beneficial to inhibitory activity, as evidenced by the NA inhibitory superiority of Sulfuretin over 4-hydroxyderricin. These inspired us to develop novel chalcone derivatives as potential NA inhibitors. Using natural Sulfuretin as the lead compound, a series of novel chalcone derivatives was designed and synthesized as potential NA inhibitors(Scheme 1). NA inhibitory activities of the synthesized compounds were evaluated in vitro, and their structure-activity relationship(SAR) was also investigated.


Fig.1 Structures of natural products 4-hydroxyderricin and Kumatakenin

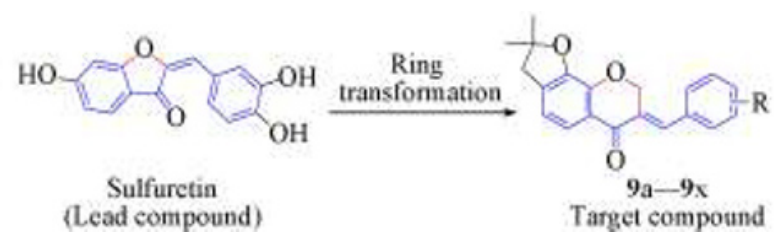

Scheme 1 Design strategy of target compounds

*Corresponding authors. Email: axhu@hnu.edu.cn; liuailin@imm.ac.cn

Received October 26, 2018; accepted January 14, 2019.

Supported by the Hunan Provincial Natural Science Foundation, China(No.2019JJ40030) and the National Natural Science Foundation of China(No.81673480).

(C) Jilin University, The Editorial Department of Chemical Research in Chinese Universities and Springer-Verlag GmbH 


\section{Experimental}

\subsection{Reagents and Instruments}

All the solvents and reagents were commercially available and used without further purification unless specified. All reactions were monitored by thin-layer chromatography(TLC) on $0.25 \mathrm{~mm}$ Huanghai GF254 silica gel coated plates. Flash column chromatography was performed on a column packed with silica gel(300-400 mesh) using petroleum ether and ethyl acetate as eluent. The ${ }^{1} \mathrm{H}$ and ${ }^{13} \mathrm{C}$ NMR spectra were recorded on a Bruker $400 \mathrm{MHz}$ Advance spectrometer using tetramethylsilane(TMS) as the internal standard. Mass spectra were obtained on a Finnigan LCQ Advantage MAX instrument with electrospray ionization(ESI-MS) method. Elemental analyses were performed with a Vario EL III(Germany) instrument.

\subsection{General Synthetic Procedure for Target Compounds 3 and 5}

To a solution of $\mathrm{NaOH}(6.0 \mathrm{mmol})$ in $\operatorname{EtOH}(20 \mathrm{~mL})$ were added compound 2 or $\mathbf{4}(2.0 \mathrm{mmol})$ and 4-hydroxybenzaldehyde ( $2.0 \mathrm{mmol}$ ). Dilute $\mathrm{HCl}$ was added to adjust the $\mathrm{pH}$ value to 2 and then the mixture was filtered. The residue was purified by column chromatography to afford corresponding compound $\mathbf{3}$ or 5.

(E)-1-(4-Hydroxy-2,2-dimethyl-2,3-dihydrobenzofuran-7yl)-3-(4-hydroxy-phenyl)prop-2-en-1-one(3): a white solid, yield 54\%, m. p. $244-245{ }^{\circ} \mathrm{C} .{ }^{1} \mathrm{H}$ NMR(400 MHz, DMSO-d 6 ), $\delta: \quad 10.29(\mathrm{~s}, 1 \mathrm{H}), 10.01(\mathrm{~s}, 1 \mathrm{H}), 7.67(\mathrm{~d}, J=15.6 \mathrm{~Hz}, 1 \mathrm{H})$, $7.60-7.49(\mathrm{~m}, 4 \mathrm{H}), 6.84(\mathrm{~d}, J=7.8 \mathrm{~Hz}, 2 \mathrm{H}), 6.43(\mathrm{~d}, J=8.6 \mathrm{~Hz}$, $1 \mathrm{H}), 2.91(\mathrm{~s}, 2 \mathrm{H}), 1.51(\mathrm{~s}, 6 \mathrm{H}) .{ }^{13} \mathrm{C} \mathrm{NMR}\left(100 \mathrm{MHz}, \mathrm{DMSO}-\mathrm{d}_{6}\right)$, $\delta$ : 185.74, 160.42, 159.66, 158.78, 141.62, 130.26, 130.11, $126.09,122.62,115.98,114.19,112.98,108.93,88.64,40.15$, 28.11. ESI-MS, $m / z: 311[\mathrm{M}+\mathrm{H}]^{+}$. Elemental anal.(\%) calcd. for $\mathrm{C}_{19} \mathrm{H}_{18} \mathrm{O}_{4}$ : C 73.53, $\mathrm{H}$ 5.85; found: C 73.56, H 5.83.

(E)-1-(7-Hydroxy-2,2-dimethyl-2,3-dihydrobenzofuran-5yl)-3-(4-hydroxy-phenyl)prop-2-en-1-one(5): a white solid, yield $58 \%$, m. p. $223-224{ }^{\circ} \mathrm{C} .{ }^{1} \mathrm{H}$ NMR(400 MHz, DMSO-d 6 ), $\delta: \quad 10.03(\mathrm{~s}, 1 \mathrm{H}), \quad 9.52(\mathrm{~s}, 1 \mathrm{H}), \quad 7.69(\mathrm{~d}, J=7.5 \mathrm{~Hz}, 2 \mathrm{H})$, $7.56-7.66(\mathrm{~m}, 3 \mathrm{H}), 7.41(\mathrm{~s}, 1 \mathrm{H}), 6.82(\mathrm{~d}, J=7.5 \mathrm{~Hz}, 2 \mathrm{H}), 3.07(\mathrm{~s}$, $2 \mathrm{H}), 1.45(\mathrm{~s}, 6 \mathrm{H}) .{ }^{13} \mathrm{C} \mathrm{NMR}\left(100 \mathrm{MHz}, \mathrm{DMSO}-\mathrm{d}_{6}\right), \delta: 187.04$, $159.76,150.65,142.93,141.57,131.37,130.65,128.36,126.03$, $118.74,117.81,115.76,115.70,88.50,42.18,27.96$. ESI-MS, $m / z: 311[\mathrm{M}+\mathrm{H}]^{+}$. Elemental anal.(\%) calcd. for $\mathrm{C}_{19} \mathrm{H}_{18} \mathrm{O}_{4}: \mathrm{C}$ 73.53, H 5.85; found: C 73.55, H 5.83.

\subsection{General Synthetic Procedure for Target Compounds 9a-9r}

A suspension of 2,2-dimethyl-2,3-dihydrobenzofuran-7-ol (50.0 mmol), acrylonitrile $(500.0 \mathrm{mmol})$, and $\mathrm{K}_{2} \mathrm{CO}_{3}(5.0 \mathrm{mmol})$ in tert-butanol $(5.0 \mathrm{mmol})$ was stirred at reflux for $48 \mathrm{~h}$. After cooling to room temperature, the reaction was quenched with $85 \%$ phosphoric acid $(4.0 \mathrm{mmol})$. The mixture was concentrated in vacuo. The residue was dissolved in $\mathrm{CH}_{2} \mathrm{Cl}_{2}$ and then the mixture was filtered and the filtrate was washed with sodium hydroxide solution and brine. The organic layer was concentrated in vacuo to give compound $\mathbf{6}$ as a white solid. A mixture of compound $\mathbf{6}(20.0 \mathrm{mmol})$ and concentrated $\mathrm{HCl}(30 \mathrm{~mL})$ was stirred at reflux for $2 \mathrm{~h}$, then poured into $100 \mathrm{~mL}$ of ice water and filtered. The precipitate was washed with brine and dried to afford compound 7 as an orange-red solid. The mixture of polyphosphoric acid(15 mL) and compound $7(10.0 \mathrm{mmol})$ was stirred at $60{ }^{\circ} \mathrm{C}$ for $1.5 \mathrm{~h}$, then $100 \mathrm{~mL}$ of ice water was added. The mixture was stirred for $0.5 \mathrm{~h}$ and then extracted with ethyl acetate. The organic phase was washed with sodium hydroxide solution and brine, dried by anhydrous sodium sulfate and concentrated in vacuo. The residue was purified via flash silica gel column chromatography to afford compound $\mathbf{8}$ as a yellow solid.

Phosphoric $\operatorname{acid}(85 \%, 8 \mathrm{~mL})$ was stirred at $80{ }^{\circ} \mathrm{C}$. Then compound $\mathbf{8}(1.5 \mathrm{mmol})$ and substituted aromatic aldehydes $(1.6 \mathrm{mmol})$ were added. The reaction mixture was added to the appropriate amount of ice water and stirred for $30 \mathrm{~min}$. The resultant mixture was extracted with ethyl acetate. The organic phase was washed with saturated sodium bicarbonate solution and saturated brine, dried over anhydrous sodium sulfate, and then filtered and then filtrate was concentrated under reduced pressure. The residue was purified by column chromatography or recrystallization from ethanol to afford the desired compounds $9 \mathrm{a}-9 \mathrm{r}$.

(E)-7-(3-Hydroxybenzylidene)-2,2-dimethyl-2,3,7,8-tetrahydro-6H-furo[3,2- $h]$ chromen-6-one(9a): a brown solid, yield $64 \%$, m. p. $204-206{ }^{\circ} \mathrm{C} .{ }^{1} \mathrm{H}$ NMR(400 MHz, DMSO-d $\left.{ }_{6}\right), \delta$ : 9.74(s, 1H), 7.65(s, 1H), 7.39(d, $J=8.0 \mathrm{~Hz}, 1 \mathrm{H}), 7.28-7.34(\mathrm{~m}$, $1 \mathrm{H}), 6.97(\mathrm{~d}, J=8.0 \mathrm{~Hz}, 1 \mathrm{H}), 6.80-6.90(\mathrm{~m}, 3 \mathrm{H}), 5.37(\mathrm{~d}, J=1.8$ $\mathrm{Hz}, 2 \mathrm{H}), \quad 3.09(\mathrm{~s}, 2 \mathrm{H}), 1.45(\mathrm{~s}, 6 \mathrm{H}) .{ }^{13} \mathrm{C} \mathrm{NMR}(100 \mathrm{MHz}$, DMSO-d $\left.{ }_{6}\right), \delta: 181.53,157.41,146.44,145.60,137.11,136.10$, 135.23, 130.98, 130.34, 122.00, 121.49, 119.21, 118.84, 117.12, $116.75,88.88,67.60,42.96,28.06$. ESI-MS, $m / z: 323[\mathrm{M}+\mathrm{H}]^{+}$. Elemental anal.(\%) calcd. for $\mathrm{C}_{20} \mathrm{H}_{18} \mathrm{O}_{4}$ : C 74.52, $\mathrm{H}$ 5.63; found: C 74.31, H 5.58.

(E)-7-(4-Hydroxybenzylidene)-2,2-dimethyl-2,3,7,8-tetrahydro-6H-furo[3,2- $h]$ chromen-6-one(9b): a yellow solid, yield 57\%, m. p. $203-205{ }^{\circ} \mathrm{C} .{ }^{1} \mathrm{H} \mathrm{NMR}\left(400 \mathrm{MHz}, \mathrm{DMSO}-\mathrm{d}_{6}\right), \delta$ : 10.14(s, 1H), 7.66(s, 1H), 7.36(d, $J=7.8 \mathrm{~Hz}, 1 \mathrm{H}), 7.33(\mathrm{~d}, J=8.0$ $\mathrm{Hz}, 2 \mathrm{H}), 6.95(\mathrm{~d}, J=7.8 \mathrm{~Hz}, 1 \mathrm{H}), 6.89(\mathrm{~d}, J=8.0 \mathrm{~Hz}, 2 \mathrm{H}), 5.39(\mathrm{~d}$, $J=1.9 \mathrm{~Hz}, 2 \mathrm{H}), 3.08(\mathrm{~s}, 2 \mathrm{H}), 1.44(\mathrm{~s}, 6 \mathrm{H}) .{ }^{13} \mathrm{C} \mathrm{NMR}(100 \mathrm{MHz}$, DMSO-d $\left._{6}\right), \delta: 181.41,159.19,146.39,145.39,137.35,135.76$, $133.03,128.02,125.15,122.15,119.12,118.68,116.03,88.79$, $67.79,42.93,28.07$. ESI-MS, $m / z: 323[\mathrm{M}+\mathrm{H}]^{+}$. Elemental anal.(\%) calcd. for $\mathrm{C}_{20} \mathrm{H}_{18} \mathrm{O}_{4}$ : C 74.52, H 5.63; found: C 74.47, H 5.61 .

(E)-7-(3-Chloro-4-hydroxybenzylidene)-2,2-dimethyl-2,3, 7,8-tetrahydro-6H-furo[3,2- $h]$ chromen-6-one $(9 \mathrm{c})$ : a yellow solid, yield $37 \%$, m. p. $188-190{ }^{\circ} \mathrm{C} .{ }^{1} \mathrm{H}$ NMR(400 MHz, DMSO- $\left._{6}\right), \delta: 10.91(\mathrm{~s}, 1 \mathrm{H}), 7.63(\mathrm{~s}, 1 \mathrm{H}), 7.52(\mathrm{~s}, 1 \mathrm{H}), 7.37(\mathrm{~d}$, $J=8.0 \mathrm{~Hz}, 1 \mathrm{H}), 7.27(\mathrm{~d}, J=8.4 \mathrm{~Hz}, 1 \mathrm{H}), 7.09(\mathrm{~d}, J=8.4 \mathrm{~Hz}, 1 \mathrm{H})$, $6.95(\mathrm{~d}, J=8.0 \mathrm{~Hz}, 1 \mathrm{H}), 5.39(\mathrm{~d}, J=1.9 \mathrm{~Hz}, 2 \mathrm{H}), 3.08(\mathrm{~s}, 2 \mathrm{H})$, 1.44(s, 6H). ${ }^{13} \mathrm{C}$ NMR(100 MHz, DMSO-d 6 ), $\delta: 181.11,155.08$, $146.72,145.72,135.83,132.80,131.13,129.65,126.52,122.36$, $120.62,119.26,118.76,117.19,116.24,88.79,67.89,43.19$, 
28.34. ESI-MS, $m / z$ : $735[2 \mathrm{M}+\mathrm{Na}]^{+}$. Elemental anal.(\%) calcd. for $\mathrm{C}_{20} \mathrm{H}_{17} \mathrm{ClO}_{4}$ : C 67.33, $\mathrm{H}$ 4.80; found: $\mathrm{C} 67.21, \mathrm{H} 4.76$.

(E)-7-(3-Bromo-4-hydroxybenzylidene)-2,2-dimethyl-2,3, 7,8-tetrahydro- $6 H$-furo[3,2- $h]$ chromen-6-one(9d): a yellow solid, yield 41\%, m. p. 206-208 ${ }^{\circ} \mathrm{C} .{ }^{1} \mathrm{H}$ NMR(400 MHz, DMSO-d $\left._{6}\right), \delta: 10.94(\mathrm{~s}, 1 \mathrm{H}), 7.64(\mathrm{~d}, J=12.3 \mathrm{~Hz}, 2 \mathrm{H}), 7.36(\mathrm{~d}$, $J=7.2 \mathrm{~Hz}, 1 \mathrm{H}), 7.30(\mathrm{~d}, J=7.6 \mathrm{~Hz}, 1 \mathrm{H}), 7.06(\mathrm{~d}, J=7.6 \mathrm{~Hz}, 1 \mathrm{H})$, 6.95(d, $J=7.2 \mathrm{~Hz}, 1 \mathrm{H}), 5.38(\mathrm{~d}, J=1.8 \mathrm{~Hz}, 2 \mathrm{H}), 3.08(\mathrm{~s}, 2 \mathrm{H})$, $1.44(\mathrm{~s}, 6 \mathrm{H}) .{ }^{13} \mathrm{C}$ NMR(100 MHz, DMSO-d $\left.\mathrm{d}_{6}\right), \delta: 181.10,156.11$, 146.73, 145.74, 135.81, 135.78, 135.75, 131.70, 129.65, 126.97, $122.39,119.28,118.77,116.86,110.24,88.79,67.90,43.22$, 28.35. ESI-MS, $m / z$ : $825[2 \mathrm{M}+\mathrm{Na}]^{+}$. Elemental anal.(\%) calcd. for $\mathrm{C}_{20} \mathrm{H}_{17} \mathrm{BrO}_{4}$ : C 59.87, $\mathrm{H} 4.27$; found: C 59.65, H 4.23.

(E)-7-(4-Hydroxy-3-iodobenzylidene)-2,2-dimethyl-2,3,7, 8-tetrahydro-6H-furo[3,2- $h$ ] chromen-6-one(9e): a yellow solid, yield $38 \%$, m. p. $215-217^{\circ} \mathrm{C} .{ }^{1} \mathrm{H}$ NMR(400 MHz, DMSO-d 6 ), $\delta: 11.02(\mathrm{~s}, 1 \mathrm{H}), 7.83(\mathrm{~s}, 1 \mathrm{H}), 7.61(\mathrm{~s}, 1 \mathrm{H}), 7.36(\mathrm{~d}, J=7.4 \mathrm{~Hz}, 1 \mathrm{H})$, $7.31(\mathrm{~d}, J=8.1 \mathrm{~Hz}, 1 \mathrm{H}), 7.00(\mathrm{~d}, J=8.1 \mathrm{~Hz}, 1 \mathrm{H}), 6.94(\mathrm{~d}, J=7.4$ $\mathrm{Hz}, 1 \mathrm{H}), 5.38(\mathrm{~d}, J=1.8 \mathrm{~Hz}, 2 \mathrm{H}), 3.07(\mathrm{~s}, 2 \mathrm{H}), 1.44(\mathrm{~s}, 6 \mathrm{H})$. ${ }^{13} \mathrm{C}$ NMR(100 MHz, DMSO-d 6 ), $\delta: 181.08,158.70,146.72$, $145.70,141.84,135.74,135.70,132.45,129.39,127.44,122.41$, 119.26, 118.75, 115.39, 88.77, 85.68, 67.88, 43.20, 28.35 . ESI-MS, $m / z: 449[\mathrm{M}+\mathrm{H}]^{+}$. Elemental anal.(\%) calcd. for $\mathrm{C}_{20} \mathrm{H}_{17} \mathrm{IO}_{4}$ : C 53.59, H 3.82; found: C 53.38, H 3.75.

(E)-7-(4-Hydroxy-3-nitrobenzylidene)-2,2-dimethyl-2,3,7, 8-tetrahydro-6H-furo[3,2- $h$ ]chromen-6-one(91): a yellow solid, yield $82 \%$, m. p. $220-222{ }^{\circ} \mathrm{C} .{ }^{1} \mathrm{H}$ NMR(400 MHz, DMSO-d 6 ), $\delta$ : 11.61(br s, 1H), 7.98(s, 1H), 7.68(s, 1H), 7.62(d, $J=8.6 \mathrm{~Hz}$, $1 \mathrm{H}), 7.37(\mathrm{~d}, J=7.8 \mathrm{~Hz}, 1 \mathrm{H}), 7.23(\mathrm{~d}, J=8.6 \mathrm{~Hz}, 1 \mathrm{H}), 6.95(\mathrm{~d}$, $J=7.8 \mathrm{~Hz}, 1 \mathrm{H}), 5.40(\mathrm{~d}, J=1.8 \mathrm{~Hz}, 2 \mathrm{H}), 3.08(\mathrm{~s}, 2 \mathrm{H}), 1.44(\mathrm{~s}, 6 \mathrm{H})$. ${ }^{13} \mathrm{C}$ NMR(100 MHz, DMSO-d $\left.\mathrm{d}_{6}\right), \delta: 181.02,153.21,146.73$, $145.79,137.64,136.98,135.95,134.81,130.97,127.75,125.48$, 122.24, 119.77, 119.30, 118.83, 88.82, 67.73, 43.19, 28.32 . ESI-MS, $m / z: 368[\mathrm{M}+\mathrm{H}]^{+}$. Elemental anal.(\%) calcd. for $\mathrm{C}_{20} \mathrm{H}_{17} \mathrm{NO}_{6}$ : C 65.39, $\mathrm{H} 4.66, \mathrm{~N} 3.81$; found: $\mathrm{C}$ 65.31, H 4.62, $\mathrm{N} 3.87$.

(E)-7-(3,4-Dihydroxybenzylidene)-2,2-dimethyl-2,3,7,8tetrahydro- $6 H$-furo-[3,2- $h$ ]chromen-6-one $(9 \mathrm{~g})$ : a yellow solid, yield $45 \%$, m. p. $214-216{ }^{\circ} \mathrm{C} .{ }^{1} \mathrm{H}$ NMR(400 MHz, DMSO-d 6 ), $\delta$ : 9.66(s, 1H), 9.26(s, 1H), 7.57(s, 1H), 7.35(d, $J=7.8 \mathrm{~Hz}, 1 \mathrm{H})$, 6.94(d, $J=7.8 \mathrm{~Hz}, 1 \mathrm{H}), 6.75-6.90(\mathrm{~m}, 3 \mathrm{H}), 5.38(\mathrm{~d}, J=1.8 \mathrm{~Hz}$, 2H), 3.07(s, 2H), 1.44(s, 6H). ${ }^{13} \mathrm{C}$ NMR(100 MHz, DMSO-d 6 ), $\delta: 181.20,148.25,146.70,145.89,145.66,137.62,135.53$, $128.11,125.78,123.78,122.54,119.23,118.63,118.23,116.34$, $88.72,68.05,43.22,28.35$. ESI-MS, $m / z: 339[\mathrm{M}+\mathrm{H}]^{+}$. Elemental anal.(\%) calcd. for $\mathrm{C}_{20} \mathrm{H}_{18} \mathrm{O}_{5}$ : C 71.00, H 5.36; found: $\mathrm{C}$ 69.73, H 5.33 .

(E)-7-(3-Methoxybenzylidene)-2,2-dimethyl-2,3,7,8tetrahydro- $6 H$-furo[3,2- $h]$ chromen-6-one( $9 \mathrm{~h})$ : a yellow solid, yield $58 \%$, m. p. $119-121{ }^{\circ} \mathrm{C} .{ }^{1} \mathrm{H} \mathrm{NMR}\left(400 \mathrm{MHz}, \mathrm{CDCl}_{3}\right), \delta$ : $7.83(\mathrm{~s}, 1 \mathrm{H}), 7.56(\mathrm{~d}, J=8.0 \mathrm{~Hz}, 1 \mathrm{H}), 7.32-7.38(\mathrm{~m}, 1 \mathrm{H})$, $6.92-6.99(\mathrm{~m}, 1 \mathrm{H}), 6.80-6.91(\mathrm{~m}, 3 \mathrm{H}), 5.39(\mathrm{~d}, J=1.8 \mathrm{~Hz}, 2 \mathrm{H})$, $3.83(\mathrm{~s}, 3 \mathrm{H}), 3.08(\mathrm{~s}, 2 \mathrm{H}), 1.54(\mathrm{~s}, 6 \mathrm{H}) .{ }^{13} \mathrm{C} \mathrm{NMR}(100 \mathrm{MHz}$, $\left.\mathrm{CDCl}_{3}\right), \delta: 181.30,159.81,146.77,145.91,136.85,135.98$, $135.66,131.62,130.34,122.84,122.33,119.34,118.86,116.02$, $115.82,88.82,67.90,55.70,43.22,28.33$. ESI-MS, $m / z$ :
$337[\mathrm{M}+\mathrm{H}]^{+}$. Elemental anal.(\%) calcd. for $\mathrm{C}_{21} \mathrm{H}_{20} \mathrm{O}_{4}: \mathrm{C} 74.98$, H 5.99; found: C 74.88, H 5.95.

(E)-7-(4-Methoxybenzylidene)-2,2-dimethyl-2,3,7,8tetrahydro-6H-furo[3,2- $h]$ chromen-6-one(9i): a yellow solid, yield $47 \%$, m. p. $132-134{ }^{\circ} \mathrm{C} .{ }^{1} \mathrm{H} \mathrm{NMR}\left(400 \mathrm{MHz}, \mathrm{CDCl}_{3}\right), \delta$ : 7.83(s, 1H), 7.56(d, $J=8.0 \mathrm{~Hz}, 1 \mathrm{H}), 7.28(\mathrm{~d}, J=8.8 \mathrm{~Hz}, 2 \mathrm{H})$, $6.97(\mathrm{~d}, J=8.8 \mathrm{~Hz}, 2 \mathrm{H}), 6.87(\mathrm{~d}, J=8.0 \mathrm{~Hz}, 1 \mathrm{H}), 5.42(\mathrm{~d}, J=1.8$ $\mathrm{Hz}, 2 \mathrm{H}), 3.86(\mathrm{~s}, 3 \mathrm{H}), 3.08(\mathrm{~s}, 2 \mathrm{H}), 1.54(\mathrm{~s}, 6 \mathrm{H}) .{ }^{13} \mathrm{C} \mathrm{NMR}(100$ MHz, DMSO-d 6 ), $\delta: 181.22,160.96,146.73,145.74,136.79$, $135.73,132.88,129.22,126.85,122.46,119.28,118.75,114.86$, 88.78, 68.01, 55.86, 43.23, 28.36. ESI-MS, $m / z: 337[\mathrm{M}+\mathrm{H}]^{+}$. Elemental anal.(\%) calcd. for $\mathrm{C}_{21} \mathrm{H}_{20} \mathrm{O}_{4}$ : C 74.98, H 5.99; found C 74.76, H 5.89.

(E)-2,2-Dimethyl-7-(3,4,5-trimethoxybenzylidene)2,3,7,8-tetrahydro-6H-furo[3,2- $h]$ chromen-6-one(9j): a brown solid, yield $46 \%$, m. p. $125-127{ }^{\circ} \mathrm{C} .{ }^{1} \mathrm{H}$ NMR(400 MHz, $\left.\mathrm{CDCl}_{3}\right), \delta: 7.79(\mathrm{~s}, 1 \mathrm{H}), 7.56(\mathrm{~d}, J=8.0 \mathrm{~Hz}, 1 \mathrm{H}), 6.89(\mathrm{~d}, J=8.0$ $\mathrm{Hz}, 1 \mathrm{H}), 6.54(\mathrm{~s}, 2 \mathrm{H}), 5.46(\mathrm{~d}, J=1.8 \mathrm{~Hz}, 2 \mathrm{H}), 3.91(\mathrm{~s}, 3 \mathrm{H})$, $3.88(\mathrm{~s}, 6 \mathrm{H}), 3.09(\mathrm{~s}, 2 \mathrm{H}), 1.54(\mathrm{~s}, 6 \mathrm{H}) .{ }^{13} \mathrm{C} \mathrm{NMR}(100 \mathrm{MHz}$, $\left.\mathrm{CDCl}_{3}\right), \delta: 181.58,153.26,146.64,145.83,139.36,137.41$, $135.00,130.20,129.84,122.36,119.73,118.28,107.42,88.69$, 68.12, 60.97, 56.19, 43.58, 28.21. ESI-MS, $m / z: 397[\mathrm{M}+\mathrm{H}]^{+}$. Elemental anal.(\%) calcd. for $\mathrm{C}_{23} \mathrm{H}_{24} \mathrm{O}_{6}: \mathrm{C} 69.68, \mathrm{H} 6.10$; found: C 69.57, H 6.07.

(E)-7-(4-Hydroxy-3-methoxybenzylidene)-2,2-dimethyl2,3,7,8-tetrahydro-6H-furo[3,2- $h$ ]chromen-6-one(9k): a yellow solid, yield 55\%, m. p. $136-138{ }^{\circ} \mathrm{C} .{ }^{1} \mathrm{H}$ NMR(400 MHz, $\left.\mathrm{CDCl}_{3}\right), \delta: 7.80(\mathrm{~s}, 1 \mathrm{H}), 7.56(\mathrm{~d}, J=8.0 \mathrm{~Hz}, 1 \mathrm{H}), 6.98(\mathrm{~d}, J=8.0$ $\mathrm{Hz}, 1 \mathrm{H}), 6.83-6.89(\mathrm{~m}, 3 \mathrm{H}), 5.87(\mathrm{br} \mathrm{s}, 1 \mathrm{H}), 5.44(\mathrm{~d}, J=1.8 \mathrm{~Hz}$, 2H), 3.92(s, 3H), 3.08(s, 2H), 1.54(s, 6H). ${ }^{13} \mathrm{C} \mathrm{NMR(100} \mathrm{MHz,}$ DMSO-d $\left._{6}\right), \delta: 181.16,149.21,148.11,146.74,145.71,137.55$, $135.59,128.49,125.78,124.91,122.56,119.24,118.68,116.17$, $115.21,88.72,68.15,56.16,43.23,28.35$. ESI-MS, $\mathrm{m} / \mathrm{z}$ : $353[\mathrm{M}+\mathrm{H}]^{+}$. Elemental anal.(\%) calcd. for $\mathrm{C}_{21} \mathrm{H}_{20} \mathrm{O}_{5}: \mathrm{C} 71.58$, H 5.72; found: C 71.46, H 5.69.

(E)-7-(3-Hydroxy-4-methoxybenzylidene)-2,2-dimethyl2,3,7,8-tetrahydro-6H-furo[3,2- $h$ ]chromen-6-one(91): a brown solid, yield $60 \%$, m. p. $194-196{ }^{\circ} \mathrm{C} .{ }^{1} \mathrm{H}$ NMR(400 MHz, DMSO-d $\left._{6}\right), \delta: 9.36(\mathrm{~s}, 1 \mathrm{H}), 7.61(\mathrm{~s}, 1 \mathrm{H}), 7.37(\mathrm{~d}, J=8.0 \mathrm{~Hz}, 1 \mathrm{H})$, $7.05(\mathrm{~d}, J=8.4 \mathrm{~Hz}, 1 \mathrm{H}), 6.96(\mathrm{~d}, J=8.0 \mathrm{~Hz}, 1 \mathrm{H}), 6.90-6.92(\mathrm{~m}$, $2 \mathrm{H}), 5.40(\mathrm{~d}, J=1.9 \mathrm{~Hz}, 2 \mathrm{H}), 3.85(\mathrm{~s}, 3 \mathrm{H}), 3.09(\mathrm{~s}, 2 \mathrm{H}), 1.45(\mathrm{~s}$, $6 \mathrm{H}) .{ }^{13} \mathrm{C}$ NMR(100 MHz, DMSO-d 6 ), $\delta: 181.19,149.58$, $146.51,146.45,145.45,137.13,135.69,128.80,126.80,123.28$, $122.18,119.11,118.65,117.32,112.33,88.72,67.77,55.89$, 42.97, 28.13. ESI-MS, $m / z: 353[\mathrm{M}+\mathrm{H}]^{+}$. Elemental anal.(\%) calcd. for $\mathrm{C}_{21} \mathrm{H}_{20} \mathrm{O}_{5}$ : C 71.58, H 5.72; found: C 71.49, H 5.70.

(E)-7-(2-Hydroxy-3-methoxybenzylidene)-2,2-dimethyl2,3,7,8-tetrahydro-6H-furo[3,2- $h$ ]chromen-6-one(9m): a yellow solid, yield $48 \%$, m. p. $178-180{ }^{\circ} \mathrm{C} .{ }^{1} \mathrm{H}$ NMR(400 MHz, DMSO-d $\left._{6}\right), \delta: 9.41(\mathrm{~s}, 1 \mathrm{H}), 7.84(\mathrm{~s}, 1 \mathrm{H}), 7.38(\mathrm{~d}, J=8.0 \mathrm{~Hz}, 1 \mathrm{H})$, $7.08(\mathrm{~d}, J=8.0 \mathrm{~Hz}, 1 \mathrm{H}), 6.93-6.97(\mathrm{~m}, 1 \mathrm{H}), 6.87(\mathrm{t}, J=8.0 \mathrm{~Hz}$, $1 \mathrm{H}), 6.70-6.73(\mathrm{~m}, 1 \mathrm{H}), 5.26(\mathrm{~d}, J=1.8 \mathrm{~Hz}, 2 \mathrm{H}), 3.84(\mathrm{~s}, 3 \mathrm{H})$, $3.08(\mathrm{~s}, 2 \mathrm{H}), 1.43(\mathrm{~s}, 6 \mathrm{H}) .{ }^{13} \mathrm{C} \mathrm{NMR}\left(100 \mathrm{MHz}, \mathrm{DMSO}_{6}\right)$, $\delta: 181.52,148.31,146.77,146.43,145.98,135.77,133.30$, $130.41,122.54,122.40,121.80,119.40,119.33,118.77,113.86$, $88.77,68.29,56.43,43.23,28.34$. ESI-MS, $m / z: 353[\mathrm{M}+\mathrm{H}]^{+}$. 
Elemental anal.(\%) calcd. for $\mathrm{C}_{21} \mathrm{H}_{20} \mathrm{O}_{5}$ : C 71.58, H 5.72; found: C 71.43, H 5.67.

(E)-7-[4-(Dimethylamino)benzylidene]-2,2-dimethyl2,3,7,8-tetrahydro- $6 H$-furo[3,2- $h]$ chromen-6-one(9n): an orange solid, yield 58\%, m. p. 202-204 ${ }^{\circ} \mathrm{C}$. ${ }^{1} \mathrm{H}$ NMR(400 MHz, DMSO-d $\left._{6}\right), \delta: 7.65(\mathrm{~s}, 1 \mathrm{H}), 7.34(\mathrm{~m}, 3 \mathrm{H}), 6.93(\mathrm{~d}, J=8.0 \mathrm{~Hz}, 1 \mathrm{H})$, 6.79(d, $J=8.4 \mathrm{~Hz}, 2 \mathrm{H}), 5.42(\mathrm{~d}, J=1.8 \mathrm{~Hz}, 2 \mathrm{H}), 3.07(\mathrm{~s}, 2 \mathrm{H})$, 3.01(s, 6H), 1.44(s, 6H). ${ }^{13} \mathrm{C}$ NMR(100 MHz, DMSO-d 6 ), $\delta$ : $180.88,151.60,146.67,145.52,137.74,135.24,133.10,126.03$, $122.75,121.63,119.16,118.52,112.22,88.65,68.34,43.23$, 40.09, 28.37. ESI-MS, $m / z: 350[\mathrm{M}+\mathrm{H}]^{+}$. Elemental anal.(\%) calcd. for $\mathrm{C}_{22} \mathrm{H}_{23} \mathrm{NO}_{3}$ : C 75.62, H 6.63, N 4.01; found: C 75.54, H 6.66, N 4.13.

(E)-4-(\{2,2-Dimethyl-6-oxo-3,6-dihydro-2H-furo[3,2- $h]$ chromen-7(8H)-ylidene -methyl)benzoic acid(9o): a yellow solid, yield $55 \%$, m. p. $274-276{ }^{\circ} \mathrm{C} .{ }^{1} \mathrm{H} \mathrm{NMR}(400 \mathrm{MHz}$, DMSO- $\left._{6}\right), \delta: 13.15($ br s, $1 \mathrm{H}), 8.03(\mathrm{~d}, J=8.0 \mathrm{~Hz}, 2 \mathrm{H}), 7.77(\mathrm{~s}$, $1 \mathrm{H}), 7.57(\mathrm{~d}, J=8.0 \mathrm{~Hz}, 2 \mathrm{H}), 7.39(\mathrm{~d}, J=8.0 \mathrm{~Hz}, 1 \mathrm{H}), 6.98(\mathrm{~d}$, $J=8.0 \mathrm{~Hz}, 1 \mathrm{H}), 5.39(\mathrm{~d}, J=1.8 \mathrm{~Hz}, 2 \mathrm{H}), 3.09$ (s, 2H), 1.44(s, 6H). ${ }^{13} \mathrm{C}$ NMR(100 MHz, DMSO-d 6 ), $\delta: 181.18,167.27,146.78$, $145.95,138.48,136.13,135.64,132.98,131.71,130.74,130.01$, 122.23, 119.38, 118.93, 88.86, 67.76, 43.22, 28.32. ESI-MS, $m / z: 351[\mathrm{M}+\mathrm{H}]^{+}$. Elemental anal.(\%) calcd. for $\mathrm{C}_{21} \mathrm{H}_{18} \mathrm{O}_{5}$ : C 71.99, H 5.18; found: C 71.90, H 5.15.

(E)-2,2-Dimethyl-7-(2-nitrobenzylidene)-2,3,7,8tetrahydro- $6 H$-furo[3,2- $h]$ chromen-6-one $(9 \mathrm{p})$ : a yellow solid, yield $78 \%$, m. p. $142-144{ }^{\circ} \mathrm{C} .{ }^{1} \mathrm{H}$ NMR(400 MHz, DMSO-d 6 ), $\delta: \quad 8.25-8.31(\mathrm{~m}, 1 \mathrm{H}), 7.93(\mathrm{~s}, 1 \mathrm{H}), \quad 7.85-7.90(\mathrm{~m}, 1 \mathrm{H})$, $7.72-7.78(\mathrm{~m}, 1 \mathrm{H}), 7.43-7.48(\mathrm{~m}, 1 \mathrm{H}), 7.42(\mathrm{~d}, J=8.0 \mathrm{~Hz}, 1 \mathrm{H})$, $7.00(\mathrm{~d}, J=8.0 \mathrm{~Hz}, 1 \mathrm{H}), 5.17(\mathrm{~d}, J=1.8 \mathrm{~Hz}, 2 \mathrm{H}), 3.10(\mathrm{~s}, 2 \mathrm{H})$, $1.44(\mathrm{~s}, 6 \mathrm{H}) .{ }^{13} \mathrm{C}$ NMR(100 MHz, DMSO-d 6 ), $\delta: 181.44,148.15$, $146.85,146.21,136.28,134.70,134.26,132.18,131.82,130.98$, $129.97,125.66,122.27,119.42,119.02,88.89,67.50,43.24$, 28.31. ESI-MS, $m / z: 352[\mathrm{M}+\mathrm{H}]^{+}$. Elemental anal.(\%) calcd. for $\mathrm{C}_{20} \mathrm{H}_{17} \mathrm{NO}_{5}$ : C 68.37, $\mathrm{H} 4.88, \mathrm{~N} 3.99$; found: $\mathrm{C} 68.28, \mathrm{H} 4.84$, N 3.92 .

(E)-2,2-Dimethyl-7-(3-nitrobenzylidene)-2,3,7,8tetrahydro- $6 H$-furo[3,2- $h]$ chromen-6-one $(9 \mathrm{q})$ : a yellow solid, yield $83 \%$, m. p. $231-233{ }^{\circ} \mathrm{C} .{ }^{1} \mathrm{H}$ NMR(400 MHz, DMSO-d 6 ), $\delta: 8.26-8.34(\mathrm{~m}, 2 \mathrm{H}), 7.79-7.89(\mathrm{~m}, 3 \mathrm{H}), 7.40(\mathrm{~d}, J=8.0 \mathrm{~Hz}$, $1 \mathrm{H}), 6.99(\mathrm{~d}, J=8.0 \mathrm{~Hz}, 1 \mathrm{H}), 5.41(\mathrm{~d}, J=1.8 \mathrm{~Hz}, 2 \mathrm{H}), 3.09(\mathrm{~s}, 2 \mathrm{H})$, $1.44(\mathrm{~s}, 6 \mathrm{H}) .{ }^{13} \mathrm{C}$ NMR(100 MHz, DMSO-d 6 ), $\delta: 181.50,147.66$, $146.79,145.98,143.13,141.07,136.60,136.27,134.51,133.60$, 130.76, 124.37, 122.16, 119.38, 119.00, 88.91, 67.54, 43.23, 28.33. ESI-MS, $m / z: 352[\mathrm{M}+\mathrm{H}]^{+}$. Elemental anal.(\%) calcd. for $\mathrm{C}_{20} \mathrm{H}_{17} \mathrm{NO}_{5}$ : C 68.37, $\mathrm{H} 4.88, \mathrm{~N} 3.99$; found: C 68.18, H 4.81, N 3.89 .

(E)-2,2-Dimethyl-7-(4-nitrobenzylidene)-2,3,7,8tetrahydro-6H-furo[3,2- $h$ ]chromen-6-one(9r): a yellow solid, yield $85 \%$, m. p. $238-240{ }^{\circ} \mathrm{C} .{ }^{1} \mathrm{H}$ NMR(400 MHz, DMSO-d 6 ), $\delta: 8.32(\mathrm{~d}, J=8.0 \mathrm{~Hz}, 2 \mathrm{H}), 7.81(\mathrm{~s}, 1 \mathrm{H}), 7.73(\mathrm{~d}, J=8.0 \mathrm{~Hz}, 2 \mathrm{H})$, $7.40(\mathrm{~d}, J=8.0 \mathrm{~Hz}, 1 \mathrm{H}), 6.99(\mathrm{~d}, J=8.0 \mathrm{~Hz}, 1 \mathrm{H}), 5.39(\mathrm{~d}, J=2.0$ $\mathrm{Hz}, 2 \mathrm{H}), 3.09(\mathrm{~s}, 2 \mathrm{H}), 1.44(\mathrm{~s}, 6 \mathrm{H}) .{ }^{13} \mathrm{C} \mathrm{NMR}(100 \mathrm{MHz}$, DMSO-d $\left._{6}\right), \delta: 181.04,147.89,146.79,146.00,140.92,136.34$, $134.42,134.28,131.73,124.19,122.13,119.41,119.05,88.93$, 67.64, 43.24, 28.33. ESI-MS, $m / z: 352[\mathrm{M}+\mathrm{H}]^{+}$. Elemental anal.(\%) calcd. for $\mathrm{C}_{20} \mathrm{H}_{17} \mathrm{NO}_{5}$ : C 68.37, H 4.88, N 3.99; found: C $68.21, \mathrm{H} 4.82$, N 3.88 .

\subsection{General Synthetic Procedure for Target Compounds 9s-9u}

A suspension of each of compounds $9 \mathrm{p}-9 \mathrm{r}(1.00 \mathrm{mmol})$ and the appropriate amount of iron powder in $\mathrm{CH}_{2} \mathrm{Cl}_{2}(10 \mathrm{~mL})$, $\mathrm{H}_{2} \mathrm{O}(1 \mathrm{~mL})$ and acetic acid $(10 \mathrm{~mL})$ was stirred at room temperature for $6.0 \mathrm{~h}$. The mixture was filtered and the residue was washed with $\mathrm{CH}_{2} \mathrm{Cl}_{2}$. The filtrate was washed with saturated sodium bicarbonate solution and brine. The organic phase was dried by anhydrous sodium sulfate, filtered and concentrated in vacuo. The residue was recrystallized by ethanol to provide compounds $9 \mathrm{~s}-9 \mathrm{u}$, respectively.

(E)-7-(2-Aminobenzylidene)-2,2-dimethyl-2,3,7,8tetrahydro-6 $H$-furo[3,2- $h]$ chromen-6-one $(9 \mathrm{~s})$ : a brown solid, yield $82 \%$, m. p. $182-184{ }^{\circ} \mathrm{C} .{ }^{1} \mathrm{H}$ NMR(400 MHz, DMSO-d 6 ), $\delta: 7.68(\mathrm{~s}, 1 \mathrm{H}), 7.39(\mathrm{~d}, J=7.8 \mathrm{~Hz}, 1 \mathrm{H}), 7.13(\mathrm{t}, J=7.5 \mathrm{~Hz}, 1 \mathrm{H})$, $6.95(\mathrm{~d}, J=7.8 \mathrm{~Hz}, 1 \mathrm{H}), 6.70-6.89(\mathrm{~m}, 2 \mathrm{H}), 6.59(\mathrm{t}, J=7.2 \mathrm{~Hz}$, $1 \mathrm{H}), 5.49$ (br s, $2 \mathrm{H}), 5.26(\mathrm{~d}, J=1.8 \mathrm{~Hz}, 2 \mathrm{H}), 3.07(\mathrm{~s}, 2 \mathrm{H}), 1.43(\mathrm{~s}$, $6 \mathrm{H}) .{ }^{13} \mathrm{C} \mathrm{NMR}\left(100 \mathrm{MHz}, \mathrm{DMSO}-\mathrm{d}_{6}\right), \delta: 181.76,148.94$, $146.77,145.81,135.57,134.76,131.42,130.55,129.80,122.67$, $119.18,118.65,118.26,116.10,115.95,88.72,68.31,43.24$, 28.34. ESI-MS, $m / z: 322[\mathrm{M}+\mathrm{H}]^{+}$. Elemental anal.(\%) calcd. for $\mathrm{C}_{20} \mathrm{H}_{19} \mathrm{NO}_{3}$ : C 74.75, $\mathrm{H}$ 5.96, N 4.36; found: C 74.55, H 5.91, $\mathrm{N} 4.41$.

(E)-7-(3-Aminobenzylidene)-2,2-dimethyl-2,3,7,8tetrahydro-6 $H$-furo[3,2- $h$ ]chromen-6-one $(9 \mathrm{t})$ : a yellow solid, yield $75 \%$, m. p. $152-154{ }^{\circ} \mathrm{C} .{ }^{1} \mathrm{H} \mathrm{NMR}\left(400 \mathrm{MHz}, \mathrm{DMSO}-\mathrm{d}_{6}\right)$, $\delta: 7.58(\mathrm{~s}, 1 \mathrm{H}), 7.37(\mathrm{~d}, J=7.8 \mathrm{~Hz}, 1 \mathrm{H}), 7.09-7.19(\mathrm{~m}, 1 \mathrm{H})$, $6.96(\mathrm{~d}, J=7.8 \mathrm{~Hz}, 1 \mathrm{H}), 6.52-6.69(\mathrm{~m}, 3 \mathrm{H}), 5.36(\mathrm{~d}, J=1.8 \mathrm{~Hz}$, $2 \mathrm{H}), 5.29$ (br s, 2H), 3.08(s, 2H), 1.44(s, 6H). ${ }^{13} \mathrm{C}$ NMR(100 MHz, DMSO-d 6 ), $\delta: 181.46,149.49,146.77,145.91,137.92$, $135.79,134.85,130.63,129.77,122.46,119.33,118.75,118.34$, $115.90,115.52,88.76,68.00,43.24,28.33$. ESI-MS, $\mathrm{m} / z$ : $322[\mathrm{M}+\mathrm{H}]^{+}$. Elemental anal.(\%) calcd. for $\mathrm{C}_{20} \mathrm{H}_{19} \mathrm{NO}_{3}: \mathrm{C} 74.75$, H 5.96, N 4.36; found: C 74.67, H 5.87, N 4.30.

(E)-7-(4-Aminobenzylidene)-2,2-dimethyl-2,3,7,8tetrahydro-6H-furo[3,2- $h]$ chromen-6-one $(9 \mathrm{u})$ : an orange solid, yield $95 \%$. m. p. $217-219^{\circ} \mathrm{C} .{ }^{1} \mathrm{H}$ NMR(400 MHz, DMSO-d 6 ), $\delta$ : 7.59(s, 1H), 7.34(d, $J=8.0 \mathrm{~Hz}, 1 \mathrm{H}), 7.19(\mathrm{~d}, J=8.5 \mathrm{~Hz}, 2 \mathrm{H})$, $6.93(\mathrm{~d}, J=8.0 \mathrm{~Hz}, 1 \mathrm{H}), 6.65(\mathrm{~d}, J=8.5 \mathrm{~Hz}, 2 \mathrm{H}), 5.95(\mathrm{br} \mathrm{s}, 2 \mathrm{H})$, $5.40(\mathrm{~d}, J=1.8 \mathrm{~Hz}, 2 \mathrm{H}), 3.08(\mathrm{~s}, 2 \mathrm{H}), 1.45(\mathrm{~s}, 6 \mathrm{H}) .{ }^{13} \mathrm{C}$ NMR $(100$ MHz, DMSO-d $\left.\mathrm{d}_{6}\right), \delta: 180.85,151.29,146.42,145.26,138.10$, $135.09,133.30,125.14,122.53,121.36,118.96,118.37,113.81$, $88.53,68.16,42.99,28.17$. ESI-MS, $m / z: 322[\mathrm{M}+\mathrm{H}]^{+}$. Elemental anal.(\%) calcd. for $\mathrm{C}_{20} \mathrm{H}_{19} \mathrm{NO}_{3}: \mathrm{C} 74.75, \mathrm{H} 5.96, \mathrm{~N} 4.36$; found: C 74.59, H 5.93, N 4.27.

\subsection{Synthetic Procedure for Target Compounds $9 \mathrm{v}-9 \mathrm{x}$}

(E)-2,2-Dimethyl-7-[4-(methylamino)benzylidene]-2,3,7, 8 -tetrahydro-6H-furo[3,2- $h]$ chromen-6-one $(9 \mathrm{v})$ : potassium carbonate $(5.0 \mathrm{mmol})$ and iodomethane $(3.0 \mathrm{mmol})$ were added to a solution of compound $9 \mathrm{u}(1.0 \mathrm{mmol})$ in dimethyl sulfoxide 
$(6 \mathrm{~mL})$. The reaction mixture was stirred for $3.0 \mathrm{~h}$ at room temperature, then diluted with water and extracted with ethyl acetate. The separated organic layer was washed with saturated brine and concentrated. The crude product was purified by column chromatography to give compound $9 \mathrm{v}$ as a yellow solid. Yield 33\%, m. p. $176-178{ }^{\circ} \mathrm{C} .{ }^{1} \mathrm{H} \mathrm{NMR}\left(400 \mathrm{MHz}, \mathrm{CDCl}_{3}\right), \delta$ : $7.80(\mathrm{~s}, 1 \mathrm{H}), 7.55(\mathrm{~d}, J=7.8 \mathrm{~Hz}, 1 \mathrm{H}), 7.21(\mathrm{~d}, J=8.0 \mathrm{~Hz}, 2 \mathrm{H})$, $6.85(\mathrm{~d}, J=7.8 \mathrm{~Hz}, 1 \mathrm{H}), 6.67(\mathrm{~d}, J=8.0 \mathrm{~Hz}, 2 \mathrm{H}), 5.46(\mathrm{~d}, J=1.8$ $\mathrm{Hz}, 2 \mathrm{H}), 4.84(\mathrm{br} \mathrm{s}, 1 \mathrm{H}), 3.07(\mathrm{~s}, 2 \mathrm{H}), 2.91(\mathrm{~s}, 3 \mathrm{H}), 1.54(\mathrm{~s}, 6 \mathrm{H})$. ${ }^{13} \mathrm{C} \mathrm{NMR}\left(100 \mathrm{MHz}, \mathrm{CDCl}_{3}\right), \delta: 181.83,149.86,146.57,145.67$, $138.14,134.45,132.54,126.62,123.88,122.64,119.64,117.95$, $112.56,88.54,68.48,43.61,30.58,28.26$. ESI-MS, $\mathrm{m} / z$ : $336[\mathrm{M}+\mathrm{H}]^{+}$. Elemental anal.(\%) calcd. for $\mathrm{C}_{21} \mathrm{H}_{21} \mathrm{NO}_{3}$ : C 75.20, H 6.31, N 4.18; found: C 75.06, H 6.28, N 4.27.

(E)- $N$-[4-( $\{2,2-D i m e t h y l-6-o x o-3,6$-dihydro- $2 H$-furo$[3,2-h]$ chromen-7(8H)-ylidene $\}$-methyl)phenyl $]$ acetamide $(9 \mathrm{w})$ : acetic anhydride $(1.0 \mathrm{mmol})$ was added to the solution of compound $9 \mathrm{u}(1.0 \mathrm{mmol})$ in acetic acid $(10 \mathrm{~mL})$. The mixture was stirred at reflux for $2.0 \mathrm{~h}$, then diluted with water and extracted with ethyl acetate. The organic phase was washed with a saturated sodium carbonate solution and saturated brine, dried by anhydrous sodium sulfate, filtered and concentrated under vacuum to yield compound $9 \mathrm{w}$ as a yellow solid. Yield $95 \%$, m. p. $234-236{ }^{\circ} \mathrm{C} .{ }^{1} \mathrm{H}$ NMR(400 MHz, DMSO-d $)$ ), $\delta$ : $10.22(\mathrm{~s}$, $1 \mathrm{H}, \mathrm{NH}), 7.72(\mathrm{~d}, J=8.0 \mathrm{~Hz}, 2 \mathrm{H}), 7.67(\mathrm{~s}, 1 \mathrm{H}), 7.42(\mathrm{~d}, J=8.0 \mathrm{~Hz}$, 2H), 7.37(d, $J=7.8 \mathrm{~Hz}, 1 \mathrm{H}), 6.95(\mathrm{~d}, J=7.8 \mathrm{~Hz}, 1 \mathrm{H}), 5.41(\mathrm{~d}$, $J=1.8 \mathrm{~Hz}, 2 \mathrm{H}), 3.08(\mathrm{~s}, 2 \mathrm{H}), 2.09(\mathrm{~s}, 3 \mathrm{H}), 1.44(\mathrm{~s}, 6 \mathrm{H}) .{ }^{13} \mathrm{C} \mathrm{NMR}$ (100 MHz, DMSO-d $\left.{ }_{6}\right), \delta: 181.21,169.20,146.74,145.77$, $141.21,136.63,135.77,131.94,129.79,128.85,122.42,119.28$, 119.22, 118.76, 88.78, 68.01, 43.22, 28.35, 24.61. ESI-MS, $\mathrm{m} / \mathrm{z}$ : $364[\mathrm{M}+\mathrm{H}]^{+}$. Elemental anal.(\%) calcd. for $\mathrm{C}_{22} \mathrm{H}_{21} \mathrm{NO}_{4}$ : C 72.71, H 5.82, N 3.85; found: C 72.62, H 5.80, N 3.79.

(E)- $N$-[4-( $\{2,2$-Dimethyl-6-oxo-3,6-dihydro- $2 H$-furo[3,2- $h]$ chromen-7(8H)-ylidene $\}$-methyl)phenyl]-methanesulfonamide $(9 \mathrm{x})$ : methanesulfonyl chloride $(1.2 \mathrm{mmol})$ was added dropwise to a stirred solution of compound $9 \mathrm{u}(1.0 \mathrm{mmol})$ and pyridine $(2.0 \mathrm{mmol})$ in $\mathrm{CH}_{2} \mathrm{Cl}_{2}(10 \mathrm{~mL})$ at ice bath. After completion of the reaction as monitored by thin layer chromography(TLC), the reaction mixture was diluted with water. The organic phase was washed with saturated brine and then dried by anhydrous sodium sulfate, filtered and concentrated under vacuum. The crude product was purified by recrystallization from ethanol to give compound $9 \mathrm{x}$ as a yellow solid. Yield $84 \%$, m. p. $210-212{ }^{\circ} \mathrm{C} .{ }^{1} \mathrm{H}$ NMR(400 MHz, DMSO-d 6 ), $\delta: 10.19$ (s, $1 \mathrm{H}), 7.68(\mathrm{~s}, 1 \mathrm{H}), 7.28-7.46(\mathrm{~m}, 5 \mathrm{H}), 6.96(\mathrm{~d}, J=7.3 \mathrm{~Hz}, 1 \mathrm{H})$, $5.40(\mathrm{~d}, J=1.8 \mathrm{~Hz}, 2 \mathrm{H}), 3.04-3.14(\mathrm{~m}, 5 \mathrm{H}), 1.44(\mathrm{~s}, 6 \mathrm{H})$. ${ }^{13} \mathrm{C}$ NMR(100 MHz, DMSO-d $\left.\mathrm{d}_{6}\right), \delta: 181.19,146.73,145.78$, $140.38,136.35,135.85,132.39,130.16,129.19,122.38$, $119.30,118.95,118.81,88.82,67.95,43.20,40.23,28.35$. ESI-MS, $m / z: 400[\mathrm{M}+\mathrm{H}]^{+}$. Elemental anal.(\%) calcd. for $\mathrm{C}_{21} \mathrm{H}_{21} \mathrm{NO}_{5} \mathrm{~S}$ : C 63.14, H 5.30, N 3.51; found: C 63.02, H 5.26, N 3.43 .

\subsection{Biological Activity Screening}

Twenty-seven synthesized compounds were tested NA inhibitory activities in vitro. NA inhibition assay was described in the literature ${ }^{[19,20]}$ with some modifications. The substrate $\quad 2$-(4-methyl-umbelliferyl)- $\alpha$ - $D$-acetyl-neuraminic acid(MUNANA) could be specifically cleaved by NA, with a fluorescent product, which was quantified to determine the NA activity. In this study, we used A/PR/8/34(H1N1) as the source of NA, which was kindly donated by the China Center for Disease Control. In the enzymatic reaction system, $30 \mu \mathrm{L}$ of NA enzyme in $32.5 \mathrm{mmol} / \mathrm{L}$ 2- $\mathrm{N}$-morpholinoethanesulfonic acid (MES) buffer( $\mathrm{pH}=6.5$ ) was first incubated with $10 \mu \mathrm{L}$ of tested compounds at different concentrations in a 96-well plate at $37{ }^{\circ} \mathrm{C}$ for $10 \mathrm{~min}$. Then, $20 \mu \mathrm{L}(20 \mu \mathrm{mol} / \mathrm{L})$ of MUNANA was added. After $60 \mathrm{~min}$ incubation at $37{ }^{\circ} \mathrm{C}$, the reaction was terminated by $150 \mu \mathrm{L}$ of $\mathrm{NaOH}(34 \mathrm{mmol} / \mathrm{L}, \mathrm{pH}=12.19)$, and then the fluorescence intensity was measured at excitation wavelength of $360 \mathrm{~nm}$ and emission wavelength of $450 \mathrm{~nm}$. There were four groups in our study, including test group(with tested compounds, H1N1 virus, and MUNANA), virus control group(with MES buffer instead of tested compounds), substrate control group(with MES buffer instead of tested compounds and $\mathrm{H} 1 \mathrm{~N} 1$ virus) and positive control(with Zanamivir, H1N1 virus, and MUNANA). The NA inhibition rate(\%) was calculated using the following equation: NA inhibition rate $(\%)=$ $\left[1-\left(F_{\text {test }}-F_{\text {control }}\right) /\left(F_{\text {virus }}-F_{\text {control }}\right)\right] \times 100 \%$. The $F_{\text {test }}, F_{\text {virus }}$, and $F_{\text {control }}$ represent the fluorescence intensity of the test group, virus group, and control group, respectively. The $\mathrm{IC}_{50}$ rate value was calculated by plotting inhibition rate versus the inhibitor concentration and determination of each point. The data are expressed as the mean of three independent experiments.

\subsection{Molecular Docking}

The cocrystal complex of H1N1 NA in complex with Zanamivir was obtained from the Protein Data Bank(PDB 3TI5). Before docking, the lowest energy conformations of the test compounds were generated by energy minimization using an MMFF94 force field. The structure of the protein was obtained using LePro. The docking studies were performed using LeDock with high speed and accuracy ${ }^{[21]}$. The number of binding poses was set to 30 and other parameters were used with their default values. The dimension of the binding pocket was set as $X_{\min }=20.0, X_{\max }=39.4, Y_{\min }=7.1, Y_{\max }=21.6, Z_{\min }=-29.9$, $Z_{\max }=-12.2$. PyMol v0.99. $\mathrm{x}^{[22]}$ was used for the visualization of NA and the docked compounds. In order to clearly show the interaction between the docked compounds and H1N1 NA, LigPlot+v1.4.5 ${ }^{[23]}$ was used to generate two-dimensional interaction diagrams.

\section{Results and Discussion}

\subsection{Synthetic Route}

The target compounds $\mathbf{3}$ and $\mathbf{5}$ were synthesized as shown in Scheme 2. Claisen rearrangement of the allyl-aryl ether $\mathbf{1}$ in aluminum isopropoxide and PEG200 afforded intermediate 2 and by-product. The preparation of compound $\mathbf{2}$ was facilitated when the reaction temperature was around $185^{\circ} \mathrm{C}$. Then compounds $\mathbf{3}$ and $\mathbf{5}$ were synthesized by the aldol condensation reaction. 



Scheme 2 Syntheses of target compounds 3 and 5

The synthetic routes of target compounds $9 \mathrm{a}-\mathbf{9} \mathrm{x}$ are shown in Scheme 3. Compound $\mathbf{6}$ was synthesized by Michael addition with acrylonitrile and the cyano-group was hydrolyzed to produce compound 7. The intermediate 8 was prepared via the intramolecular Friedel-Crafts acylation. Aldol condensation of intermediate $\mathbf{8}$ with aromatic aldehydes afforded compounds 9a-9r. In addition, compounds $9 \mathrm{p}-9 \mathrm{r}$ were reduced to afford amino compounds $9 \mathrm{~s}-\mathbf{9 u}$. Compound $9 \mathrm{v}$ was synthesized by $\mathrm{N}$-methylation. Compound $9 \mathrm{w}$ was obtained by the full acetylation of the amino group. Finally, compound $9 \mathrm{x}$ was produced by sulfonylation.



9a: R=3-OH; 9b: R=4-OH; 9c: R=4-OH-3-Cl; 9d: R=4-OH-3-Br; 9e: R=4-OH-3-I; 9f: R=4-OH-3-NO ${ }_{2}$; 9g: R=3,4-(OH)

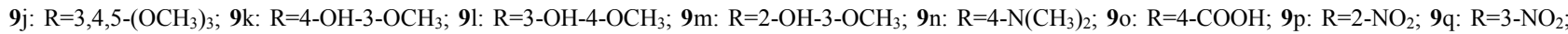

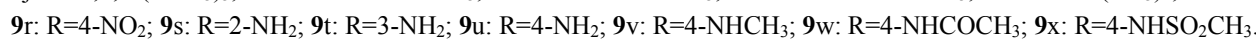

\section{Scheme 3 Synthetic routes of target compounds 9a-9x}

\subsection{Crystal Structure Analysis}

Compound 9a was cultivated in anhydrous ethanol to give a yellow transparent single crystal. The single crystal data(CCDC No.1567543) analysis showed that the crystal belongs to monoclinic system, space group $C 2 / c, a=1.72596(5)$, $b=0.68543(7), c=2.68501(7) \mathrm{nm}, Z=8, V=3.16190(1) \mathrm{nm}^{3}$, $M_{\mathrm{r}}=336.39, \quad D_{\mathrm{c}}=1.354 \quad \mathrm{Mg} / \mathrm{m}^{3}, \quad S=1.074, \quad \mu=0.766 \mathrm{~mm}^{-1}$, $F(000)=1360, T=150(2) \mathrm{K}$ and $3.307^{\circ} \leqslant \theta \leqslant 67.002^{\circ}$. The molecule is made up of two planes consisting of the benzene ring and furochromanone moiety(Fig.2). The dihedral angle between the benzene ring and furochroman-one moiety is $38.4^{\circ}$. The bond length of $\mathrm{C} 11=\mathrm{O} 3$ is $0.1221 \mathrm{~nm}$, and the bond length of $\mathrm{C} 12=\mathrm{C} 14$ generated by the condensation reaction is $0.1343 \mathrm{~nm}$. The whole molecule is present in the form of geometric $E$ isomer and consistent with the results of nuclear magnetic resonance. In addition, the entire crystal structure is stabilized via van der Waals forces.



Fig.2 Crystal structure of compound 9a

\subsection{Biological Activity and Structure-activity Re- lationship}

The synthesized compounds were evaluated for NA inhibitory activity(Table 1) in vitro. Based on the evaluation results, the SARs of tested compounds were discussed. The initial screening results showed that most of the compounds possessed excellent inhibitory activities. Then, fourteen compounds identified with high inhibition rates(over 50\%) were selected for further evaluation NA inhibitory activity and the $\mathrm{IC}_{50}$ values are listed in Table 1. The evaluation results showed that compound $9 \mathrm{~b}$ had the best NA inhibitory activity with an $\mathrm{IC}_{50}$ value of $25.31 \mu \mathrm{mol} / \mathrm{L}$.

Compound 9a showed better NA inhibitory activity than intermediate 8. This indicated that aromatic ketone had weak effect on NA inhibitory activity, but the effects of chalcone system and functional groups substituted on the phenyl ring were significant. To further explore, single or multiple functional groups on the phenyl ring were replaced. Compounds 9a-9g and 9k-9m, with hydroxyl substituents, had better NA inhibitory activities than the others. Compounds 9b, 9c and 9k with the hydroxy substituent at the para position of benzene ring had better NA inhibitory activity than compounds $9 \mathrm{a}, 91$ and $9 \mathrm{~m}$ with the hydroxy substituent at the ortho or meta position of the benzene ring. Compounds $9 \mathrm{~h}$ and $9 \mathrm{i}$, in which alkyl groups were introduced showed weak NA inhibitory activities. The NA inhibitory activities were significantly decreased when halogen $(\mathrm{Cl}, \mathrm{Br}$ and $\mathrm{I})$, nitro and methoxy groups $(\mathbf{9} \mathrm{c}-\mathbf{9} \mathrm{f}$ and $9 \mathrm{k})$ were introduced on the phenol ring. It can be explained by 
the steric hindrance effect. Unfortunately compounds $9 \mathrm{p}-9 \mathrm{r}$ with a nitro group at the ortho, meta or para position of the benzene ring displayed poor NA inhibitory activities. In contrast, there was a significant increase in NA inhibitory activities for compounds $9 \mathrm{~s}-9 \mathrm{u}$ when nitro groups were reduced to amino groups. Furthermore, the effect of substituents $4-\mathrm{NHCH}_{3}$, 4- $\mathrm{NHCOCH}_{3}$ and 4- $\mathrm{NHSO}_{2} \mathrm{CH}_{3}$ were also discussed, but compounds $9 \mathrm{v}-9 \mathrm{x}$ showed weak NA inhibitory activities. Compound 9o with the carboxyl group at para position showed moderate NA inhibitory activity. In order to investigate the effect of pyran ring on NA inhibitory activity, chalcone derivatives $\mathbf{3}$ and $\mathbf{5}$ were synthesized and evaluated for NA inhibitory activity. The results showed that compounds $\mathbf{3}$ and $\mathbf{5}$ had moderate NA inhibitory activities, similar to the natural Sulfuretin and 4-hydroxyderricin. But the NA inhibitory activities of compounds $\mathbf{3}$ and $\mathbf{5}$ were weaker than compound $\mathbf{9 b}$ with pyran ring, indicating that the pyran ring was important for the improvement of NA inhibitory activity.

Table 1 NA inhibitory activities of target compounds in vitro

\begin{tabular}{|c|c|c|c|}
\hline Compound & $\mathrm{R}$ & Inhibition rate $(\%)$ & $\mathrm{IC}_{50} /\left(\mu \mathrm{mol} \cdot \mathrm{L}^{-1}\right)$ \\
\hline 3 & - & $69.49 \pm 2.05$ & $63.03 \pm 3.32$ \\
\hline 5 & - & $76.92 \pm 0.91$ & $48.30 \pm 2.16$ \\
\hline 8 & - & $17.69 \pm 3.13$ & - \\
\hline 9a & $3-\mathrm{OH}$ & $60.49 \pm 12.20$ & $91.39 \pm 30.03$ \\
\hline $9 \mathrm{~b}$ & $4-\mathrm{OH}$ & $79.15 \pm 4.86$ & $25.31 \pm 2.48$ \\
\hline 9c & $4-\mathrm{OH}-3-\mathrm{Cl}$ & $80.39 \pm 1.29$ & $51.37 \pm 0.84$ \\
\hline 9d & 4-OH-3-Br & $74.57 \pm 1.86$ & $51.34 \pm 2.27$ \\
\hline $9 \mathrm{e}$ & 4-OH-3-I & $74.77 \pm 2.16$ & $48.68 \pm 1.70$ \\
\hline $9 \mathrm{f}$ & $4-\mathrm{OH}-3-\mathrm{NO}_{2}$ & $53.94 \pm 1.62$ & $97.10 \pm 3.84$ \\
\hline $9 \mathrm{~g}$ & $3,4-(\mathrm{OH})_{2}$ & $72.24 \pm 0.29$ & $58.78 \pm 8.25$ \\
\hline $9 \mathrm{~h}$ & $3-\mathrm{OCH}_{3}$ & $18.46 \pm 2.38$ & - \\
\hline $9 \mathrm{i}$ & $4-\mathrm{OCH}_{3}$ & $8.03 \pm 2.04$ & - \\
\hline $9 \mathrm{j}$ & 3,4,5-triOCH 3 & $28.69 \pm 1.74$ & - \\
\hline $9 \mathrm{k}$ & $4-\mathrm{OH}-3-\mathrm{OCH}_{3}$ & $94.43 \pm 3.24$ & $38.91 \pm 2.24$ \\
\hline 91 & $3-\mathrm{OH}-4-\mathrm{OCH}_{3}$ & $65.84 \pm 2.86$ & $71.03 \pm 6.38$ \\
\hline $9 \mathrm{~m}$ & $2-\mathrm{OH}-3-\mathrm{OCH}_{3}$ & $78.87 \pm 0.87$ & $76.31 \pm 20.18$ \\
\hline $9 n$ & $4-\mathrm{N}\left(\mathrm{CH}_{3}\right)_{2}$ & $35.58 \pm 18.59$ & - \\
\hline 9o & 4-COOH & $73.21 \pm 3.48$ & $76.35 \pm 13.07$ \\
\hline $9 p$ & $2-\mathrm{NO}_{2}$ & $13.45 \pm 3.19$ & - \\
\hline $9 \mathrm{q}$ & $3-\mathrm{NO}_{2}$ & $27.28 \pm 3.61$ & - \\
\hline 9r & $4-\mathrm{NO}_{2}$ & $29.8 \pm 4.56$ & - \\
\hline 9s & $2-\mathrm{NH}_{2}$ & $39.43 \pm 3.87$ & - \\
\hline $9 \mathrm{t}$ & $3-\mathrm{NH}_{2}$ & $41.9 \pm 6.67$ & - \\
\hline $9 \mathrm{u}$ & 4- $\mathrm{NH}_{2}$ & $67.89 \pm 9.85$ & $60.18 \pm 4.54$ \\
\hline $9 \mathrm{v}$ & $4-\mathrm{NHCH}_{3}$ & $13.03 \pm 2.13$ & - \\
\hline $9 \mathrm{w}$ & 4-NHCOCH 3 & $6.73 \pm 1.34$ & - \\
\hline $9 x$ & $4-\mathrm{NHSO}_{2} \mathrm{CH}_{3}$ & $6.27 \pm 1.65$ & - \\
\hline Sulfuretin & - & - & $29.6 \pm 0.5^{a}$ \\
\hline Zanamivir & - & $92.17 \pm 1.07^{b}$ & $2.86 \pm 0.36^{c}$ \\
\hline
\end{tabular}

$a$. The $\mathrm{IC}_{50}$ value of Sulfuretin is from ref.[16]; $b$. Zanamivir at a concentration of $0.004 \mu \mathrm{g} / \mathrm{mL} ; c$. in $\mathrm{nmol} / \mathrm{L}$.

In summary, compounds $9 \mathrm{~b}, \mathbf{9 o}$ and $9 \mathrm{u}$ with hydroxy, amino and carboxyl groups at the para position had better NA inhibitory activity than the others. Compound $9 \mathrm{~b}$ had higher potent NA inhibitory activity than the natural compound sulfuretin, indicating that conversion of a five-membered furan ring to a six-membered pyran ring is advantageous for the improvement of NA inhibitory activity. As shown in Fig.3, it is significant that the substituent containing hydrogen bond donor at the para position is beneficial to the NA inhibitory activities. With the same substituent, NA inhibitory activity: 4-position> 3-position>2-position; when the substituents are not identical, $4-\mathrm{OH}$ has the best NA inhibitory activity, and $\mathrm{OH}>\mathrm{NH}_{2}>$ $\mathrm{COOH}>\mathrm{N}\left(\mathrm{CH}_{3}\right)_{2}>\mathrm{NO}_{2}>\mathrm{OCH}_{3}$; halogen has no significant effect on NA inhibitory activity. And the experimental results indicated that the NA inhibitory activities were reduced when the hydroxy and amino groups were methylated, acetylated or sulfonylated, indicating that substituent with hydrogen bond donor at para position and chalcone systems are important for NA inhibitory activity. Compound $\mathbf{9 b}$ can be used as a novel lead compound for further structural optimization and the development of NA inhibitors.

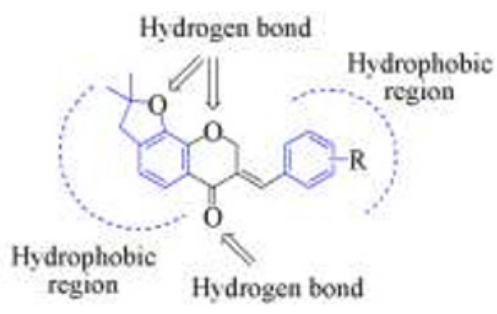

Fig.3 SAR of target compounds

\subsection{Molecular Docking Studies}

In order to understand the effect of the possible binding interactions of compounds $\mathbf{9 b}$ and $\mathbf{9 u}$ with NA, molecular docking simulations were performed. The docking results show that compound 9b[Fig.4(A)] and 9u[Fig.4(B)] are well accommodated in SA-Cavity and have similar binding sites as Zanamivir. And compound 9b[Fig.4(C)] forms five hydrogen bonds at the NA active site. The hydroxyl group interacts with the NA active site by hydrogen bonds with Ser179 and Glu227. The carbonyl group interacts with the amino residue Arg118 by a stable hydrogen-bond. And the oxygen atoms on the heterocycle of compound $9 \mathrm{~b}$ can form two stable hydrogen bonds
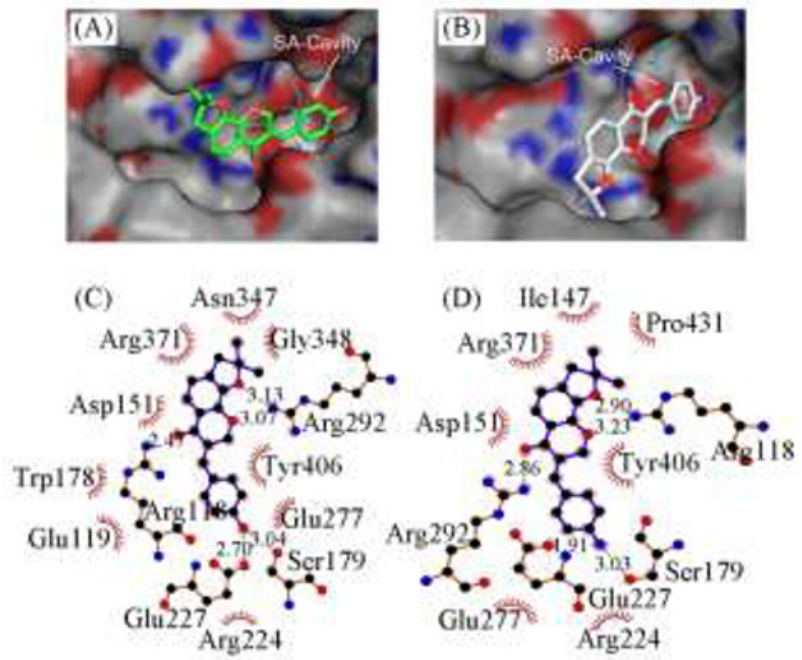

Fig.4 Interaction of compounds $9 \mathrm{~b}(\mathrm{~A}, \mathrm{C})$ and $9 u(B, D)$ with NA

Binding pockets of NA and compound $9 \mathrm{~b}$ (A) and NA and compound $9 \mathrm{u}(\mathrm{B})$ and hydrophobic and hydrogen bonding interactions between NA and compound $9 \mathrm{~b}(\mathrm{C})$ or $9 \mathrm{u}(\mathrm{D})$. 
with the amino residue Arg292. Besides, the hydrophobic interaction is formed between compound $9 \mathrm{~b}$ and nine amino acid residues(Glu119, Asp151, Trp178, Arg224, Glu277, Asn347, Gly348, Arg371 and Tyr406) of NA. As shown in Fig.4(D), five hydrogen bonds are formed between compound $9 \mathrm{u}$ and NA. The amino group binds to the NA active site by hydrogen bond interaction with Ser179 and Glu227. The carbonyl group forms a hydrogen bond with residue Arg292. And the oxygen atoms on the heterocycle interacts with the amino residue Arg118 by two hydrogen bonds. In addition, the hydrophobic interaction is formed between compound $9 \mathrm{u}$ and seven amino acid residues(Ile147, Asp151, Arg224, Glu277, Arg371, Tyr406 and Pro431) of NA. This indicates that the hydroxyl and amino groups are important for the NA inhibitory activities.

\section{Conclusions}

A series of novel chalcone derivatives containing furan or/and pyran ring was designed, synthesized and evaluated for their NA inhibitory activity in vitro. The single crystal X-ray diffraction study of compound 9 a revealed that the molecule is present in the form of geometric $E$ isomer. Among the tested compounds, compound $9 \mathrm{~b}$ showed the strongest inhibitory activity against $\mathrm{NA}$ with an $\mathrm{IC}_{50}$ value of $25.31 \mu \mathrm{mol} / \mathrm{L}$. The SAR studies revealed that the chalcone system and hydrogen bond donor substituent at the para position of phenyl ring(such as hydroxy, amino and carboxyl groups) are significant to keep excellent NA inhibitory activities. Moreover, the study results manifested that the introduction of alkyl, acyl and sulfonyl groups should be avoided in hydroxy, amino and carboxyl groups of the benzylidene moiety. On the basis of potent in vitro inhibitory activity, compound $9 \mathrm{~b}$ can be selected as a novel lead compound for further structural modification to develop novel NA inhibitors against influenza virus.

\section{References}

[1] Zhu Q., Bang T. H., Ohnuki K., Sawai T., Sawai K., Shimizua K., Sci.
Rep., 2015, 5, 13194

[2] Mills C. E., Robins J. M., Lipsitch M., Nature, 2004, 432(7019), 904

[3] Wang Y., Lei F., Li X. L., He Y., Li J., Qiu R., Wu X. Y., Hai L., Wu Y., Chem. Res. Chinese Universities, 2015, 31(6), 9421

[4] Subbarao K., Joseph T., Nat. Rev. Immunol., 2007, 7, 267

[5] De Clercq E., Nat. Rev. Drug Discov., 2006, 5(12), 1015

[6] Moscona A., N. Engl. J. Med., 2009, 360, 953

[7] Liu D., Fan Z., Jiang J., Wei J., Xin J., Chem. Res. Chinese Universities, 2013, 29(4), 706

[8] Sidwell R. W., Smee D. F., Expert Opin. Investig. Drugs, 2002, 11, 859

[9] Palese P., Nat. Med., 2004, 10(12), 82

[10] Shi F. Y., Fang H., Xu W. F., Chem. Res. Chinese Universities, 2016, 32(1), 28

[11] Ghedin E., Sengamalay N. A., Shumway M., Zaborsky J., Taylor J., Lipman D. J., Fraser C. M., Taubenberger J. K., Salzberg S. L., Nature, 2005, 437, 1162

[12] Chen B., Wang Y., Guo H., Zeng G., Eur. J. Med. Chem., 2016, 109, 199

[13] Newman D. J., Cragg G. M., J. Nat. Prod., 2016, 79(3), 629

[14] Cragg G. M., Newman D. J., BBA-Gen. Subjects, 2013, 1830(6), 3670

[15] Newman D. J., Cragg G. M., J. Nat. Prod., 2012, 75(3), 311

[16] Liu A., Wang H., Lee S. M., Wang Y., Du G., Bioorg. Med. Chem., 2008, 16, 7141

[17] Park J. Y., Jeong H. J., Kim Y. M., Park S. J., Rho M. C., Park K. H., Ryu Y. B., Lee W. S., Bioorg. Med. Chem. Lett., 2011, 21, 5602

[18] Ryu Y. B., Kim J. H., Park S. J., Chang J. S., Rho M. C., Bae K. H.,Park K. H., Lee W. S., Bioorg. Med. Chem. Lett., 2010, 20(3), 971

[19] Zu M., Yang F., Zhou W., Liu A., Du G., Zheng L., Antivir. Res., 2012, 94(3), 217

[20] Zhang J., Wang Q., Fang H., Xu W., Liu A., Du G., J. Med. Chem., 2008, 16(7), 3839

[21] Zhao H., Huang D., Caflisch A., Chem. Med. Chem., 2012, 7, 1983

[22] DeLano W. L., PyMOL, 2002, http://www. pymol.org

[23] Wallace A. C., Laskowski R. A., Thornton J. M., Protein Eng., 1995, 8,127 